\title{
Myopia disease mouse models: a missense point mutation (S673G) and a protein-truncating mutation of the Zfp644 mimic human disease phenotype
}

\author{
Katarzyna I. Szczerkowska1, Silvia Petrezselyova ${ }^{1,2}$, Jiri Lindovsky², Marcela Palkova², Jan Dvorak1, \\ Peter Makovicky², Mingyan Fang 3,4,5, Chongyi Jiang 4,5, Lingyan Chen ${ }^{4,5}$, Mingming Shi ${ }^{4,5}$, Xiao Liu 4, \\ Jianguo Zhang ${ }^{4,5}$, Agnieszka Kubik-Zahorodna ${ }^{2}$, Bjoern Schuster ${ }^{2}$, Inken M. Beck ${ }^{2,6}$, Vendula Novosadova ${ }^{2}$, \\ Jan Prochazka ${ }^{1,2}$ and Radislav Sedlacek ${ }^{1,2^{*}}$ (1)
}

\begin{abstract}
Zinc finger 644 (Zfp644 in mouse, ZNF644 in human) gene is a transcription factor whose mutation S672G is considered a potential genetic factor of inherited high myopia. ZNF644 interacts with G9a/GLP complex, which functions as a H3K9 methyltransferase to silence transcription. In this study, we generated mouse models to unravel the mechanisms leading to symptoms associated with high myopia. Employing TALEN technology, two mice mutants were generated, either with the disease-carrying mutation (Zfp644 ${ }^{5673 G}$ ) or with a truncated form of Zfp644 (Zfp644 $\left.{ }^{\triangle 8}\right)$. Eye morphology and visual functions were analysed in both mutants, revealing a significant difference in a vitreous chamber depth and lens diameter, however the physiological function of retina was preserved as found under the high-myopia conditions. Our findings prove that ZNF644/Zfp644 is involved in the development of high-myopia,

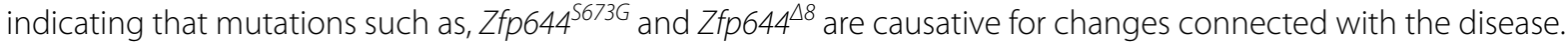
The developed models represent a valuable tool to investigate the molecular basis of myopia pathogenesis and its potential treatment.
\end{abstract}

Keywords: Myopia, Mouse model, Genetics, Zinc finger 644, Vision, Eye

\section{Introduction}

Myopia, the most common vision-related disease, is caused by a refractive error [1-3] based on elongation of the axial length of eyes, i.e. by an enlargement of vitreous and anterior chambers and by thinning of lenses but not by retinal dysfunction [1-6]. It is estimated that by the year 2020 myopia will affect 2.5 billion people [3]. The prevalence is increasing over the last few decades $[7,8]$. Myopia is dependent on multiple factors such as

\footnotetext{
*Correspondence: radislav.sedlacek@img.cas.cz

${ }^{1}$ Laboratory of Transgenic Models of Diseases, Institute of Molecular Genetics CAS, Prumyslova 595, Vestec, 25250 Prague, Czech Republic Full list of author information is available at the end of the article
}

environmental influences, educational level, diet, or genetics [2, 4, 5, 9-12].

Recently, new genetic factors have been identified to be involved in myopia development [2, 13, 14], calling for new models mimicking the human disease. Among them, ZNF644 was identified as a potential factor causing inherited myopia in different populations [13, 15-18]. Biological functions and the meaning of mutations found in ZNF644 are still unclear. So far, twelve mutations have been reported in ZNF644 to be involved in high myopia in humans, a majority of them are localised in exon three [16]. ZNF644 is a protein that binds to G9a (euchromatic histone-lysine $N$-methyltransferase 2, EHMT2) as a part of H3K9 methylation complex together with GLP (euchromatic histone-lysine $N$-methyltransferase 
1, EHMT1) [19]. It was shown that ZNF644 and WIZ, another zinc finger protein (Widely-Interspaced Zinc Finger-Containing Protein), interact with G9a and GLP complex respectively. WIZ and ZNF644 are responsible for targeting the G9a/GLP complex to specific DNA loci are also crucial for the regulation of G9a function during transcription [20]. It was also shown that ZNF644 in complex with G9a is present at Active Replication Forks. Knockdown of ZNF644 in a cell culture results in reduced cell proliferation and higher sensitivity to replication stress as well as an increase of DNA damage in replicating cells [21]. Studies in fish showed that ZNF644 with G9a/GLP complex are responsible for histone methylation critical for gene silencing during neuronal differentiation in retinal neuron differentiation [22].

Examination of myopia in humans is established and employs methods such as optical coherence tomography (OCT), electroretinography (ERG) or ultrasonography (USG) [23-26]. However, investigation of signs of myopia in mouse models is challenging due to the size of eyes [14, 27-29]. It was shown that a 5.4-6.5 $\mu \mathrm{m}$ change in axial length corresponds to one diopter change in refractive error in the eyes of C57BL/6 mice [30]. Change in ocular axial length as small as $100 \mu \mathrm{m}$ shows high myopia in C57BL/6 mice, thus the selected examination method must be very precise. In this work, we used ophthalmologic examinations known from human medical practice adapted for ophthalmological examinations of large animals [31, 32] and mice [33, 34].

Altogether, we developed and characterized two mouse mutant models of Zpf644. Zfp644 ${ }^{\mathrm{S} 673 \mathrm{G}}$ that mimics the mutation S672G found in human and Zfp644 ${ }^{\Delta 8}$, which produces a truncated protein product due to a termination codon at position AA673. We experimentally demonstrated that S673G mutation in Zfp644 is causative of a myopia phenotype and showed that large changes in Zpf644, such as protein truncation, causes a more severe phenotype. All these results point to the important regulatory role of ZNF644 in myopia development. Both Zfp644 mutant models offer new genetic tools for depicting molecular regulatory pathways involved in development of myopia and may shed more light on its potential treatment.

\section{Materials and methods}

\section{Models generation}

All animal models and experiments used in this study were ethically reviewed and performed in accordance with European directive 2010/63/EU and were approved by the Czech Central Commission for Animal Welfare.

TALENs targeting exon 3 of $Z f p 644$ gene were designed using TAL Effector Nucleotide Targeter 2.0 (https://talent.cac.cornell.edu/) [35, 36] design tool and assembled using Golden Gate cloning method. Left TALENs were designed with 16 RVDs (NN NN NI NG HD NI NI NN HD NG HD NI HD NI NN NG) followed by 16 nt spacer region and right TALENs with 15 RVDs (NN NG NN NN HD HD NN HD NG NG NI NG NN NI NI NI NG). Both TALEN plasmids were used for production of TALEN encoding mRNA as described previously [37].

TALENs mRNA was mixed with a synthetic oligodeoxynucleotide encoding mutated Zfp644 sequence ( $5^{\prime}$ AGG ATGCTAAACGGACATTTGGATCATCCAGCCAGA GCGGTAACTTCAGCAAGTTCCACAAGAGACCAC ATAGAATACAAAAAGCCCGG $3^{\prime}$ ). Targeting constructs were microinjected into male pronuclei of zygotes from C57BL/6N mice. Two lines of transgenic animals were obtained: $1 / \mathrm{Zfp} 644^{\mathrm{S} 673 \mathrm{G}}$ with desired mutation and $2 / \mathrm{Zfp} 644^{\Delta 8}$ allele with the frame shift mutation leading to a STOP codon. The animals were further maintained on C57BL/6N background. For genotyping DNA extracted from tails of 3 weeks old C57BL/6N mice using the Quick Extract DNA Extraction Solution 1.0 Kit (Illumina, USA) was used as template for PCR with following primers (forward primer for Zfp644 ${ }^{\Delta 8} 5^{\prime}$-ATCAAGCTCACA GTCAAGTAATTTT-3'; forward primer for Zfp644 ${ }^{\text {S673G: }}$ 5'-TCAGCAAGTTCCACAAGAGACC-3'; reverse primer for both alleles: $5^{\prime}$-TTGTTGGTCAGTGCTGCT CTTAAC-3').

\section{Histology}

The mice were euthanized by cervical dislocation. Eyes were sampled immediately, inserted into labelled histological cartridges, fixed in Davidson's solution for $24 \mathrm{~h}$, put into $70 \%$ ethanol solution to process using an automated tissue processor (Leica ASP 6025, Leica Microsystems, Germany), and embedded in paraffin blocks using a Leica EG $1150 \mathrm{H}$ paraffin embedding station (Leica Microsystems, Germany). Sections of 3-5 $\mu \mathrm{m}$ were cut using a microtome (Leica RM2255, Leica Microsystems, Germany) on standard glass slides (Waldemar Knittel, $\mathrm{GmbH}$, Germany). Eye samples were cut under the stereomicroscope view control and only medial cuts with optic nerve were selected for morphometry. Sections were stained with haematoxylin-eosin and mounted using Ventana Symphony H\&E Slide Stainer (Ventana Medical Systems, Inc., USA). The second set of samples were cut and fixed on salinized slides (Thermo Scientific, USA) and used for immunohistochemical procedures.

\section{RNA in situ hybridization}

Digoxygenin-labeled RNA probes (DIG RNA labeling Kit, Roche, Germany) for In Situ Hybridization (ISH) were generated by in vitro transcription from plasmid contained fragment of murine Zfp644. Procedure were carried out according to standard protocol [38] on E9.5 
after fixation in 4\% PFA in whole-mount and $2 \mu \mathrm{m}$ paraffin section for E12.5 and E14.5. Solutions used: Blocking solution (Roche, Germany), DIG-antibody (Roche, Germany), FastRed (SigmaAldrich, USA), DAPI mounting media (Molecular Probes, USA). For fluorescence and bright-field imaging, Zeiss ImagerZ2 (Zeiss, Germany) was used, for whole-mount Zeiss Apotome (Zeiss, Germany) was used.

\section{Morphometric analysis}

Samples were evaluated using a light-microscopic images obtained using a Carl Zeiss Axio Scope A1 (Zeiss, Germany) and the Axio Scan Z1 slide scanner (Zeiss, Germany).

\section{qPCR analysis}

RNA was isolated and used as a template for reverse transcription into cDNA with M-MLV Reverse Transcriptase (Promega, USA). Quantative PCR (qPCR) reactions were performed using the TATAA SYBR ${ }^{\circledR}$ GrandMaster $^{\circledR}$ Mix (TATAA Biocenter Sweden) in Cycler LightCycler ${ }^{\circledR} 480$ Instrument II (Roche, Germany). Expression levels of the genes of interest were normalized to levels of Hprt1 and Ppia and are presented as levels relative to wild type control. Primers were designed and ordered from (TATAA Biocenter), sequences are available upon request. All experiments were performed independently in triplicates on 3 different specimens $(n \geq 3)$ per group.

\section{Optical coherence tomography (OCT)}

Both retinal fundi of $15 \mathrm{Zfp} 644^{\mathrm{S} 673 \mathrm{G}}$ and $21 \mathrm{Zfp} 644^{\Delta 8}$ homozygous mice with 25 respective controls were examined. All animals were 16 weeks old. All mice were anaesthetized with $20 \%$ Zoletil-tiletamin $0.03 \mathrm{~g} / \mathrm{kg}$ and zolazepam $0.03 \mathrm{mg} / \mathrm{g}$ (Virbac, France). Pupils of eyes were dilated using eye-drops Atropin-POS 0.5\% (Ursapharm, Czech Republic). To prevent the corneal dehydration, the aqueous eye gel Vidisic $1 \times 10 \mathrm{mg}$ (Dr. Gerhard Mann Pharma, Germany) was applied on the eyes and subsequently, PMMA contact lens (Cantor\&Nissel, UK) were placed on the eyes. For the image acquisition of the retinal fundus, optical coherence tomography (OCT Spectralis ${ }^{\mathrm{TM}}$ Plus, HRA Spectralis System Heidelberg Engineering $\mathrm{GmbH}$, Heidelberg, Germany) with a $30^{\circ}$ lens was used. Mice were placed on a platform fixed in front of the OCT camera and the eye horizontally directed toward the camera; the fundus was focused and crosssectional images were taken. The segmentation of retinal layers, retinal thickness, optic disc position and blood vessels pattern were analyzed from the high-resolution cross-sectional images using HRA/Spectralis Calculation Data Manager. The average of retinal thickness was calculated from values measured in the medial cross-section in the distance of $2 \mathrm{~mm}$ to the nasal and temporal side of fundus from the optic disc (Additional file 1: Figure S2D).

\section{Electroretinography (ERG)}

ERG was performed under general anaesthesia as described above. Animals were kept on a heating pad at $37{ }^{\circ} \mathrm{C}$ with eyes protected against drying by applying transparent eye gel (Vidisic, Bausch + Lomb, Czech Republic). All measurements were done on the right eye $10 \mathrm{~min}$ after application of $0.5 \%$ solution of atropin (Ursapharm, Czech Republic). Animals were adapted to darkness for $12 \mathrm{~h}$ (over night) prior to the experiment. When the scotopic part of the stimulation protocol terminated, the mice were exposed to white background light $\left(25 \mathrm{~cd} / \mathrm{m}^{2}\right)$ at least for $2 \mathrm{~min}$ before start of the photopic stimulation protocol. The ERG stimulation and recording setup (RETI-port for animal, Roland Consult, Germany) allowed single-flash stimuli to be applied to the whole retina by ganzfeld equipped with LED diods and Xenon lamp, luminances were logarithmically distributed between 0.003 and $100 \mathrm{~cd} \mathrm{~s} / \mathrm{m}^{2}$. A golden ring (3 $\mathrm{mm}$ in diameter) was placed on the cornea as the active electrode, a golden wire inserted in the animal's mouth served as the reference electrode. Each stimulus was repeated 7-10 times and an averaged signal was saved. The signal was band-pass filtered between 1 and $300 \mathrm{~Hz}$ and digitized with $2 \mathrm{kHz}$ sampling frequency. The scotopic and photopic responses were inspected offline using a custom-made script in Matlab (MathWorks), a-wave parameters were measured in the original recording whereas b-wave parameters were quantified after removal of the oscillatory potentials from the recordings by low-pass filtering with $80 \mathrm{~Hz}$ cut-off frequency.

\section{Ultrasound imaging (USG)}

Ophthalmologic ultrasound measurements were performed on 12-14 weeks old mice. Ultrasound imaging was acquired by a Vevo 2100 Imaging System (FUJIFILM VisualSonics, Inc., Toronto, ON, Canada) equipped with a MS-550S transducer operating at a center frequency of $44 \mathrm{MHz}$. The MS550S has axial resolution of $40 \mu \mathrm{m}$ at its focal depth and allows revealing both the anterior and posterior structures of the mouse eye (Fig. 3a). For ophthalmic ultrasound imaging, mice were anesthetized with $1-2.5 \%$ isoflurane in oxygen $(1 \mathrm{~L} / \mathrm{min})$ and body temperature was maintained at $37{ }^{\circ} \mathrm{C}$. Care was taken to place the subjects in similar postures to ensure similar orientation. Sterile hypoallergenic ultrasound gel without any air bubbles was applied between the eye and the transducer and subsequently eyes were imaged. The eye structures were measured by manually delineating margins using Vevo ${ }^{\circledR}$ LAB V1.7.0. Software. The software then calculated the corresponding length of each eye. 


\section{Western blotting}

Organs were collected from adult male mice and homogenized using beads in Tissue Lyzer II (Qiagen, Germany) in NETN400 Lysis buffer (0.5\% NP-40, $50 \mathrm{mM}$ Tris- $\mathrm{HCl}$ pH 8.0, 2 mM EDTA, $400 \mathrm{mM} \mathrm{NaCl}, 10 \mathrm{mM} \mathrm{NaF}, 50 \mathrm{mM}$ $\beta$-glycerophosphate) containing protease inhibitors. Protein lysates were centrifuged at $4{ }^{\circ} \mathrm{C}$ at $3000 \mathrm{rpm}$ for $5 \mathrm{~min}$. The supernatant was carefully removed and pellets containing large plasma membrane pieces, DNA and nucleoli were diluted with NETNO Lysis buffer (no containing salts) to $100 \mathrm{mM} \mathrm{NaCl}$. Protein concentration of nuclear fractions were determined by Pierce BCA Protein Assay Kit (Thermo Scientific, USA). Samples were loaded on $8 \%$ SDS-PAGE gel and transferred onto nitrocellulose membrane (GE Healthcare Life Science, Germany). Membranes were blocked for at least $1 \mathrm{~h}$ in $5 \%$ milk in TBS-T before incubating overnight at $4{ }^{\circ} \mathrm{C}$ with the appropriate primary antibody. Antibodies used were antiZNF644 and anti-GAPDH (G9545, Sigma-Aldrich, USA). The anti-ZNF644 (raised against N-terminus AA50-602) was kindly provided by Xiaochun Yu. The following day, membranes were washed with TBS-T, incubated with appropriate secondary antibody (Sigma-Aldrich, USA) at room temperature for $1 \mathrm{~h}$ and then washed again with TBS-T. Membranes were developed using Pierce ${ }^{\mathrm{TM}}$ ECL Western Blotting substrate (Thermo Scientific ${ }^{\mathrm{TM}}$, USA) and images captured using a ChemiDoc ${ }^{\text {TM }}$ detection system (Bio-Rad).

\section{Statistical analyses}

Statistical analysis from ultrasonography examination was performed using GraphPad Prism software version 7.0 (GraphPad, USA); data was analyzed with one-way ANOVA. Data from OTC examination was performed in $\mathrm{R}$ software version 3.3 ( $\mathrm{R}$ Core Team, Austria) using linear mixed model. Data from qPCR was analyzed using Genex 6.1 (MultiD, Sweden); qPCR statistic, analysis and graphs was performed in $\mathrm{R}$ software version 3.3 ( $\mathrm{R}$ Core Team, > Austria).

\section{Results}

\section{Zfp644 is expressed in developing and adult mouse eye}

To investigate the expression pattern of Zfp644 in embryonic development, we performed whole-mount ISH of mouse embryos at E9.5 (early development of mouse eye) and fluorescence ISH of mouse embryos at E12.5 (ocular tissues differentiation) and E14.5 (corneal development). The embryonic eyes exhibited a strong hybridization signal (Fig. 1a, c, d). We also noticed strong expression in the brain and in a cervical part of a developing nervous system but not in proximal nor distal parts of spinal cord (Fig. 1a). This study was performed also on adult eyes, showing the signal in the retina and lens (Fig. 1e, e"). The level of Zfp644 expression in adult eyes was quantified in samples isolated from male and female eyes by qRT-PCR, which showed very interesting gender dependent pattern with a higher expression level in male eyes (Fig. 1h).

\section{Generation of Zfp644 mutant models}

Due to a high similarity between murine and human nucleotide sequences (Fig. 1g), the first model mimics S672G mutation in ZNF644 gene (S673G in mouse), described previously in a patient with the mutation suggested to be causative of inherited high myopia in humans (Fig. 2a). To generate the model, TALEN nucleases (TALENs) were used to specifically targeted the region in $\mathrm{Zfp} 644$ gene in combination with singlestranded oligodeoxynucleotide (ssODN) carrying the desired mutation (Fig. 2a). In addition to the insertion of ssODN with the point mutation, we also obtained a mutant with deletion of eight amino acids resulting in formation of STOP codon at position AA673, which leads to a truncation of $Z f p 644$ (Fig. 2b). To confirm a formation of truncated form of the Zfp644 protein we performed a Western Blot analysis with anti-ZNF644 antibody (Fig. 2c). The expected size of Zfp644 protein is $145 \mathrm{kDa}$, as showed in Fig. 2c, a band this size was missing in all examined organs from Zfp $644^{\Delta 8}$ animals. Both mutations were confirmed by sequencing also in $\mathrm{G} 1$ and cDNA of adult mice (Fig. 2d).

\section{Mutations in Zfp644 lead to myopia caused by enlargement of the optical axis}

Myopia is a vision-related disease caused by the elongation of the axial length of an eye. To investigate the impact of Zfp644 mutations on multiple ocular parameters in vitro we employed an ultrasound imaging technique. High-frequency ultrasound imaging [39-42] was used to image posterior structures of the eye, and in particular, the retina and optical nerve was performed with the MS-550S transducer using an imaging depth of 6 to $7 \mathrm{~mm}$ with axial resolution of $40 \mu \mathrm{m}$, thus providing a satisfied visibility for posterior eye features (Fig. 3c and Additional file 2: Figure S1). Ultrasound images of eyes with depicted ocular parameters measured in this study i.e. axial length (AL), vitreous chamber depth (VCD), lens diameter (LD) and lens thickness (LT) are shown in Fig. 3c. Ophthalmic ultrasound examinations were performed on homozygous and heterozygous $\mathrm{Zfp} 644^{\mathrm{S} 673 \mathrm{G}}$ mice (Fig. 3a), Zfp644 ${ }^{\Delta 8}$ mice (Fig. 3b), and the corresponding control (WT) mice at the age of 12-14 weeks.

In Zfp644 ${ }^{\mathrm{S} 673 \mathrm{G}}$ animals (Fig. 3a and Additional file 3: Table S1), a significant difference in optical axis enlargement was observed only in males and, more strikingly, heterozygote constitution showed higher penetrance of a phenotype than homozygote males suggesting weak 

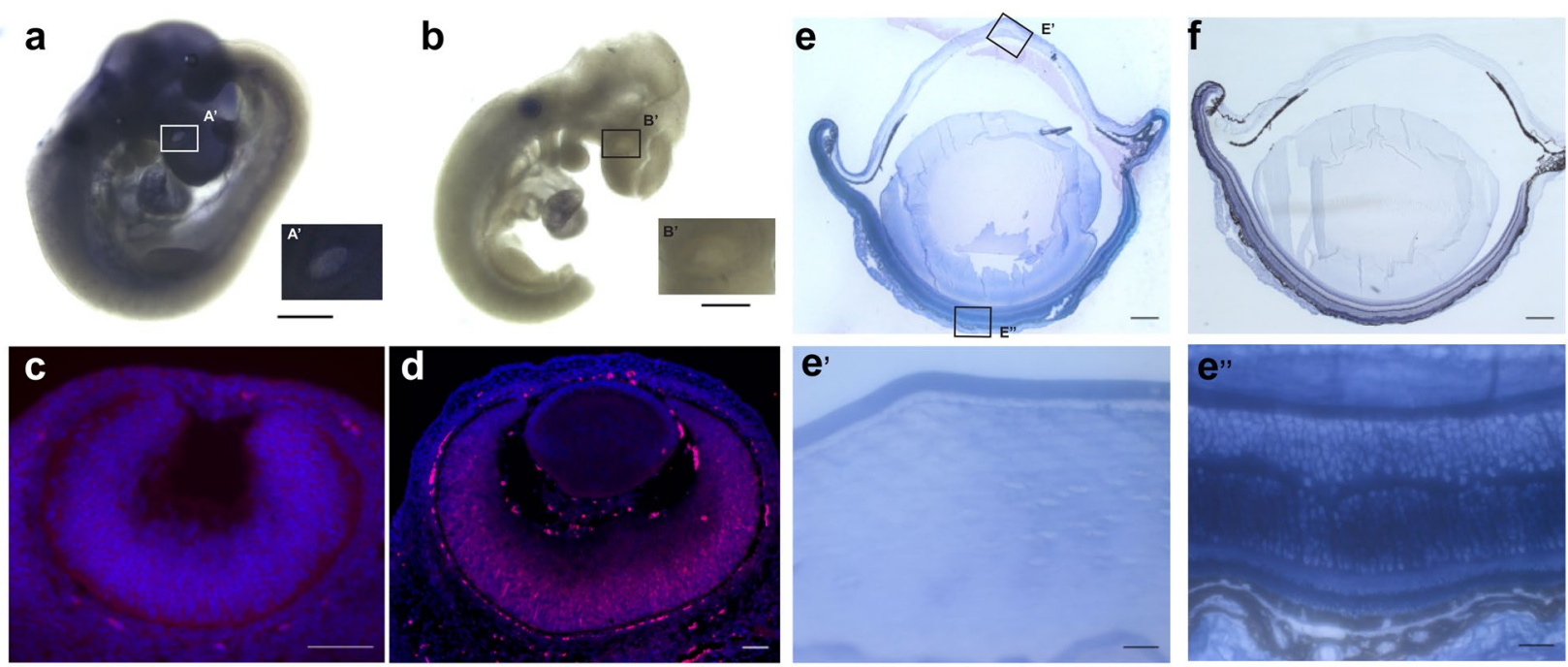

g

HUMAN ZFP644

${ }_{659 A}$ TFPKNSALKQD V KRTFGS T SOSS ${ }^{*}$ FSK I KRPHR|QKARK SIAQSG ${ }_{630 \mathrm{AA}}$

\section{MOUSE Zfp644}

660AA TFPKNSALKQD A KRTFGS S SOSS ${ }^{*} \mathrm{~N}$ FSK F HKRPHR|QKARK SIAQSG ${ }_{625 \mathrm{AA}}$

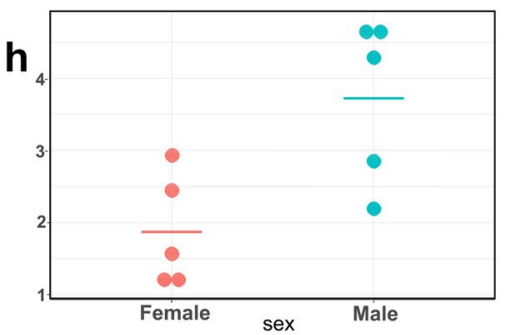

Fig. 1 Zfp644 is expressed in mouse embryonic and adult eye. a Expression of Zfp644 gene in embryonic eye at E9.5,; scale bar, 500 um. ( $A^{\prime}$ ) Focus on eye $\mathbf{b}$ Negative control; E9.5; scale bar, $500 \mu \mathrm{m}$. (B') Focus on eye $\mathbf{c}$ Expression of Zfp644 gene in eye of E 12.5; scale bar, $50 \mu \mathrm{m}$. $\mathbf{d}$ Expression of

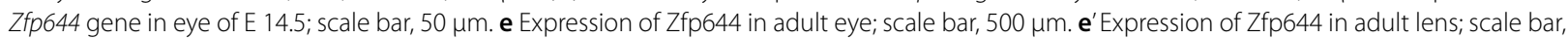
$100 \mu \mathrm{m}$. $\mathbf{e}^{\prime \prime}$ Expression of Zfp644 in adult retina scale bar, $100 \mu \mathrm{m}$. f Negative control; adult eye; $\mathbf{g}$ Protein sequence alignment of human ZNF644 and mouse Zfp644 showing position of the single point mutation at the amino acid at position 673 (asterisk); $\mathbf{h}$ Expression of Zfp644 gene in eyes of WT animals
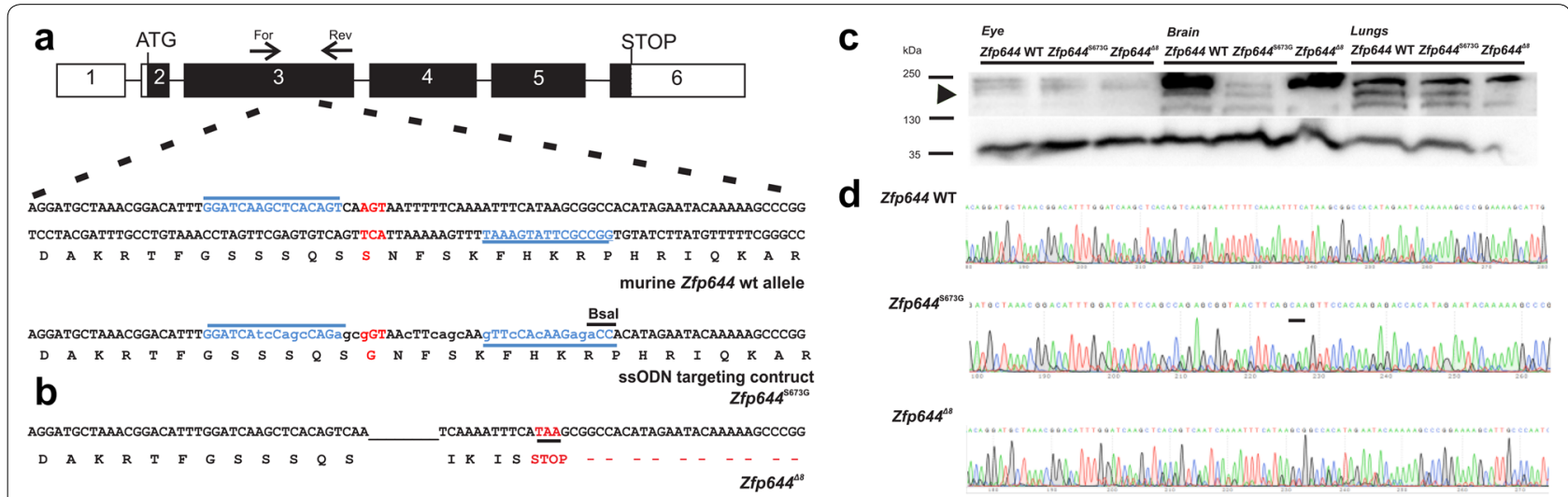

Fig. 2 Generation of Zfp644 mutant mice. a Schematic representation of murine Zfp644 gene with depiction of the targeted DNA sequence. Position of critical AGT nucleotides (red) in the exon 3 of murine Zfp644. TALEN binding sites are marked with blue underline, position of primers for PCR screening (For, Rev) are denoted. b Sequence of $Z f p 644^{\triangle 8}$ with highlighted deletion of 8 nucleotides (black underline) and a STOP codon (red letters) is shown. c In every examined tissue of Zfp644 ${ }^{\triangle 8}$ animals no detectable protein expression of Zfp644 can be found, while the samples of Zfp644 $4^{573 G}$ and control animals show protein expression in every examined tissue. Black arrow indicates correct size of Zfp644 protein (145 kDa). Expression of GAPDH protein was used as a reference protein. $\mathbf{d}$ Both mutations were confirmed by sequencing in both G1; and CDNA of adult mice eyes, brain and lungs of transgenic and control animals 


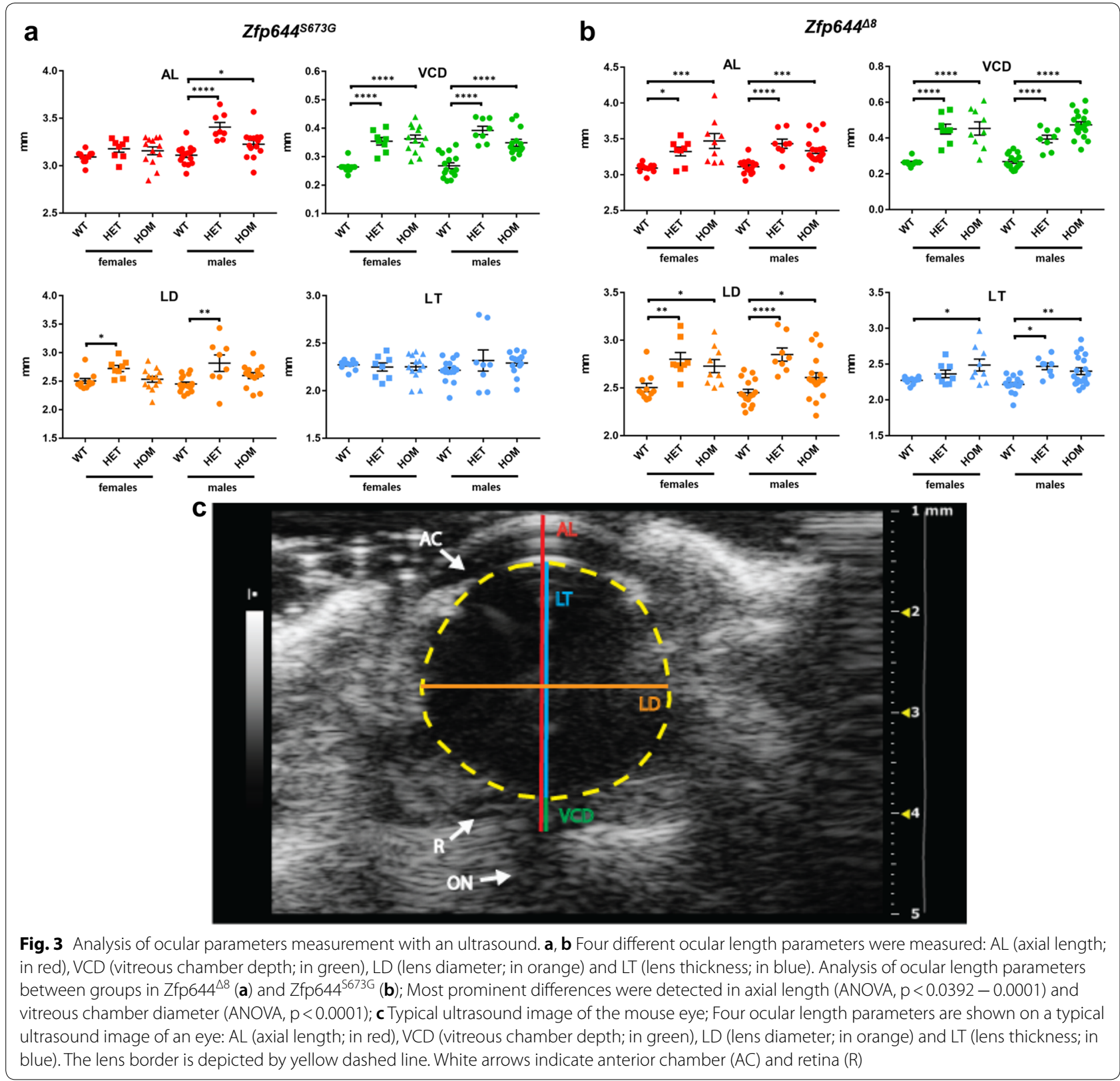

dominant negative effect of S673G mutation with gender specific bias. Nevertheless, despite no significant difference was found in total axial length in females, a vitreous chamber depth was significantly different in both, the heterozygous and homozygous animals, without gender influence. Similar to Zfp644 ${ }^{\Delta 8}$ animals, changes in lens diameter were most significant in heterozygous animals.

In Zfp644 ${ }^{\Delta 8}$ animals, alteration of the axial length is caused by morphological changes in vitreous chamber depth as well as in lens thickness and diameter (Fig. 3b and Additional file 4: Table S2). Our analysis showed that every component of the optical axis is affected by the mutation. Differences in individual ocular parameters result in enlargement of the optical axis, which results in a/the high myopia phenotype. A significant difference in all measured parameters between control and mutated mice was observed besides only one exception, the lens thickness in heterozygous females from Zfp $644^{\Delta 8}$ mice, which showed no significant difference. However, a tendency towards a high median value in heterozygous animals was evident.

In summary the protein-truncated mutation, Zfp644 ${ }^{\Delta 8}$, caused a more severe phenotype then the point mutation, which is visible in both sexes and affects also 
heterozygotes. In contrast, S673G is less penetrant and more pronounce in males, however mild changes leading to optical axis enlargement were observed also in females. Interestingly, in the case of S673G mutation, the eye morphology was affected more in heterozygous constitution in multiple parameters. Altogether, these results suggest dominant negative behavior of the mutated form of Zfp644 and closely mimics the situation reported in human patients [13].

\section{Morphology and function of the retina remains unchanged in the mutant models}

As showed previously in the fish model, ZNF644 exhibited a severe impact on retinal function and morphology
[20]. To confirm these findings, we examined morphology and function of retina in mouse Zfp644 mutants. Both eyes of Zfp644 ${ }^{\mathrm{S673G}}(\mathrm{n}=15)$ and Zfp644 ${ }^{\Delta 8}(\mathrm{n}=21)$ homozygous mutants at an age of 16 weeks were examined with OCT and compared with 25 age-matched wildtype controls. Retinal layer segmentation, optic disc position and blood vessel patterning was assessed. None of these parameters showed significant differences (Additional file 1: Figure S2A-C'). Histopathological examination of retinal structure also showed no significant difference (Fig. 4a-c'). However, in vivo imaging by OCT across the optic disc showed a significant difference in retinal thickness between control and transgenic animals (Additional file 1: Figure S2D-F). The distribution

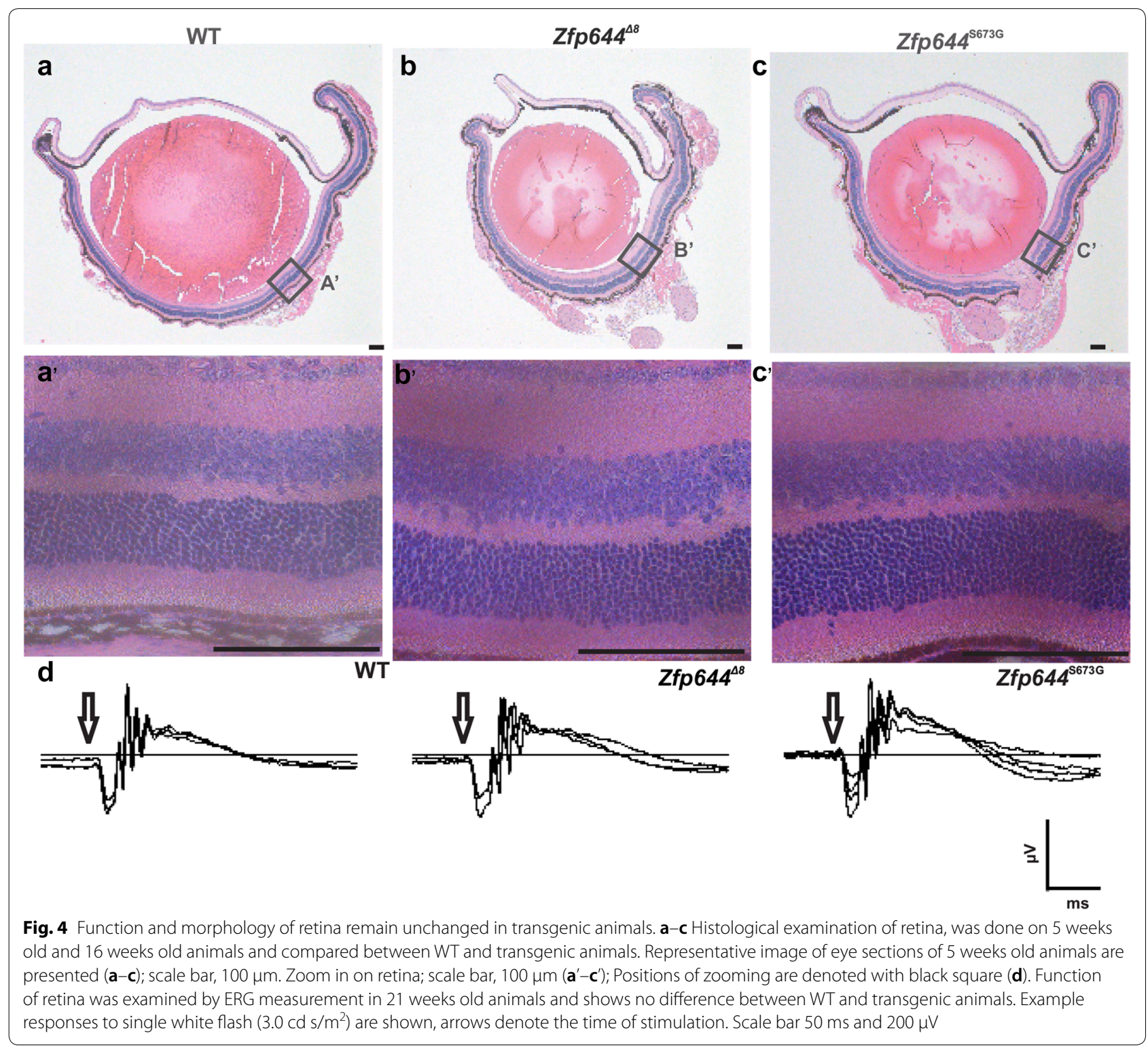


of retinal thickness in transgenic animals showed a thinner retina than in control animals. However, examination of retinal thickness on morphology sections, of different sections of retina, did not show a significant difference, but a tendency in Zfp644 ${ }^{\Delta 8}$ animals remain the same as difference found in OCT analysis (Additional file 1: Figure S2G). Overall, retinal thickness analysis showed a tendency to thin between control and transgenic animals. In order to provide functional validation of morphological findings we conducted electrophysiological measurements to assess the response of photoreceptors and other neurons to light stimulation by ERG. Amplitudes and implicit times of scotopic and photopic wave a (response of photoreceptors) and wave b (response of bipolar cells) respectively, were measured, however no abnormalities were found in the mutants (Fig. $4 \mathrm{~d}$ and Additional file 5: Figure S4A-B). In addition, quantification of cell numbers in individual retina layers (ganglion cells, outer layer cells and inner layer cells) did not reveal any significant difference (Additional file 6: Figure S3A-D). These results suggest very low or no impact of Zpf644 on the function and morphology of retina.

\section{Discussion}

Only a limited number of genetic mouse models of myopia have been generated so far. Nob ${ }^{\text {null }}$ mice, first described as a model for human night blindness [43], were found to be susceptible for a form of deprivation myopia [44-46]. Other models such as Aplp2-deficient mice [47], $P 3 h 2^{n / n}$ [48] or lumican transgenic mouse model [14] mimic the patient's phenotype only partially. Although, myopia can be studied in animals with disease induced during mouse life (e.g. by googles, no developmental, molecular nor genetic factor can be investigated $[49,50])$.

In this study, we generated two novel mouse models Zfp644 ${ }^{\mathrm{S} 673 \mathrm{G}}$ and $\mathrm{Zfp} 644^{\Delta 8}$ that conclusively mimic human inherited high myopia. We showed that both mouse models carry signs of a myopia and the phenotype is stronger in mice with truncated Zfp644 $\left(\mathrm{Zfp}_{4} 44^{\triangle 8}\right)$ than in point mutation. Moreover, we also showed that measured ocular parameters are significantly different in heterozygote animals when compared to WT animals, which corresponds with genetic conditions reported previously $[13,17]$.

No Zfp644-deficient mouse model has been generated so far, and only a fish model deficient for ZNF644 was previously described [22]. To investigate the molecular mechanism of ZNF644, Olsen et al. [22] prepared two different morphant models (MO) of $z n f 644$, based on two $z n f 644$ isoforms (a and b) whose phenotype was severe and included changes in developing retina, midbrain, and eye size. Both znf644a-MO and znf644b-MO showed signs of microphthalmia and disrupted midbrain morphology. Nevertheless, we could not reveal significant differences in retinal morphology, cell number in the retinal layers or in the electrophysiological responses of the retina, in any of $Z f p 644$ mutant mouse models.

An interesting feature of mouse Zfp644 $4^{\mathrm{S} 673 \mathrm{G}}$ mutation is not only recapitulation of human disease but there is also stronger phenotype manifestation in males. The mechanism of this phenomenon is not easy to explain but it could be a result of a different basal level of $Z f p 644$ expression in male and female eyes. The candidacy of Zfp644 in steroid hormones signaling is also supported by the work of Davis [51], in which upregulated Zfp644 expression was observed in 8 week-old ovariectomized mice following treatment with estradiol, a steroidal sex hormone.

This could also suggest potential role of steroid hormones signaling in regulation of ZNF644, although published human case reports suggested no gender specificity in any of the mutation variants of ZNF644 [13, 15-17].

Taking in account that myopia is not a retina related disease, the mouse model provides better opportunities to study the molecular role of ZNF644 in human patients with inherited high myopia, then lower vertebrate model. Therefore, genetically modified mouse models presented in this study, are advantageous mammalian models to study genetic regulations causing inherited high myopia in humans and might serve as development base for testing of potential novel therapeutic strategies.

\section{Additional files}

Additional file 1: Figure S2. Typical view of the fundus with the optic disc and blood vessels in the WT (A), Zfp644 ${ }^{\mathrm{S} 673 \mathrm{G}}$ (B) and Zfp644 ${ }^{\Delta 8}$ (C). The white spot is a reflected light. A typical view of the retinal cross-sections thru the optic disc, respectively $\left(\mathbf{A}^{\prime}-\mathbf{C}^{\prime}\right)$. ILM - internal limiting membrane, BM - Bruch's membrane. Scale bar; $200 \mu \mathrm{m}$. (D) Retinal thickness profile; position of the optic nerve is marked with a red arrow, green arrows indicate a distance in temporal and nasal retina were the retinal thickness values were collected. Retinal thickness was measured as an average of five measurements between green arrows on both temporal and nasal parts of fundus, starting from 0,5 $\mu \mathrm{m}$ from the middle of the optic disc, thru the nasal or temporal retina in 1,5 $\mu$ m distance. (E) Thickness of the retina measured on morphological sections is showed in a box plot. (F) Linear distribution of the retinal thickness is showed in a plot. (G) Statistical analyses of retinal thickness distribution are showed in a box plot.

Additional file 2: Figure S1. Representative USG image of mice eyes. Both males and females eyes of every examined group are presented.

Additional file 3: Table S1. Summary of the results of ophthalmologic ultrasound measurements on WT, HET, and HOM Zfp644 $4^{\text {S673G } 8}$ eyes. For each sex, medians, first and third quartile and p-values were calculated by one-way ANOVA analysis comparing WT, HET and HOM eyes.

Additional file 4: Table S2. Summary of the results of ophthalmologic ultrasound measurements on WT, HET, and HOM Zfp644 ${ }^{\triangle}$ eyes. For each 
sex, medians, first and third quartile and p-values were calculated by oneway ANOVA analysis comparing WT, HET and HOM eyes.

Additional file 5: Figure S4. Electroretinography. Whole-field electroretinography was recorded in animals adapted to darkness (scotopic condition) and the same animals adapted to light background (photopic condition). A) Example responses obtained in three different animals, Zfp644 WT, left column, Zfp644 S673G, middle column, and Zfp644 $\Delta 8$, right column, respectively. The time of light flash is marked with vertical dotted lines. Responses represent an averaged signal of seven to ten successive stimulations. B) Amplitude of the responses, top row, and their implicit time, bottom row, summerized for all animals and all flash luminances used. Circles represent result obtained in individual animals, lines show the mean values of each genotype, $n=4$ (Zfp644 WT), $n=3$ (Zfp644 $\mathrm{S} 673 \mathrm{G}), \mathrm{n}=5(\mathrm{Zfp} 644 \triangle 8)$. Neither the response of photoreceptors, as represented by the a-wave parameters, left column, nor the response of ON type of bipolar cells represented by b-wave, middle and right column, was significantly different between WT and mutated animals.

Additional file 6: Figure S3. Evaluations of retina cell numbers. Cells were counted in $200 \mu \mathrm{m}$. Four measurements are showed here: (A) cells in outer layer; (B) cells in inner layer; (C) ganglion cells; (D) and a total cells number. No significant differences were found.

\section{Authors' contributions}

$J D$, JP, and RS designed and supervise the study, and prepared manuscript. KS, $S P, J L, M P, P M, A K Z, B S$ and IMB performed the experiments; MF was involved in experimental design and data analysis; CJ, LC, MS, XL, and JZ performed data analysis. VN performed statistics analysis. All authors read and approved the final manuscript.

\section{Author details}

${ }^{1}$ Laboratory of Transgenic Models of Diseases, Institute of Molecular Genetics CAS, Prumyslova 595, Vestec, 25250 Prague, Czech Republic. ${ }^{2}$ Czech Centre for Phenogenomics, Institute of Molecular Genetics CAS, Prague, Czech Republic. ${ }^{3}$ Division of Clinical Immunology, Department of Laboratory Medicine, Karolinska Institutet at Karolinska University Hospital Huddinge, Stockholm, Sweden. ${ }^{4}$ BGI-Shenzhen, Shenzhen 518083, China. ${ }^{5}$ China National GeneBank, BGl-Shenzhen, Shenzhen 518120, China. ${ }^{6}$ Animal Research Center, Ulm University, Ulm, Germany.

\section{Acknowledgements}

We are grateful to Attila Juhasz, Jan Kucera, Petr Macek and members of the Transgenic and Archiving Module of CCP for excellent technical assistance and to Miles J. Raishbrook for proofreading the article.

\section{Competing interests}

The authors declare that they have no competing interests, either financial or nonfinancial, that could be perceived as prejudicing the impartiality of the research reported.

\section{Availability of data and materials}

Datasets were generated or analyzed during the current study. Data are available from the corresponding author on reasonable request.

\section{Consent for publication}

The content of the manuscript has been approved by all the authors.

\section{Ethics approval and consent to participate}

All animal models and experiments used in this study were ethically reviewed and performed in accordance with European directive 2010/63/EU and were approved by the Czech Central Commission for Animal Welfare.

\section{Funding}

The study was supported by the grant LH14276 (LH—KONTAKT II), by LM2015040 (Czech Centre for Phenogenomics), CZ.1.05/2.1.00/19.0395 ('Higher quality and capacity for transgenic models'), CZ.1.05/1.1.00/02.0109 (BIOCEV - Biotechnology and Biomedicine Centre of the Academy of Sciences and Charles University), LQ1604 (National Sustainability Program II project BIOCEV-FAR) funded by the Ministry of Education, Youth and Sports,
19-21696S by the Czech Science Foundation and the European Regional Development Fund and by RVO 68378050 by Academy of Sciences of the Czech Republic.

\section{Publisher's Note}

Springer Nature remains neutral with regard to jurisdictional claims in published maps and institutional affiliations.

Received: 18 January 2019 Accepted: 1 February 2019

Published online: 21 February 2019

\section{References}

1. Lopes MC, Andrew T, Carbonaro F, Spector TD, Hammond CJ. Estimating heritability and shared environmental effects for refractive error in twin and family studies. Invest Ophthalmol Vis Sci. 2009;50(1):126-31.

2. Young TL, Metlapally R, Shay AE. Complex trait genetics of refractive error. Arch Ophthalmol. 2007;125(1):38-48.

3. Wojciechowski R. Nature and nurture: the complex genetics of myopia and refractive error. Clin Genet. 2011;79(4):301-20.

4. Morgan IG, Ohno-Matsui K, Saw SM. Myopia. Lancet. 2012;379(9827):1739-48.

5. Hawthorne FA, Young TL. Genetic contributions to myopic refractive error: insights from human studies and supporting evidence from animal models. Exp Eye Res. 2013;114:141-9.

6. Pararajasegaram R. VISION 2020-the right to sight: from strategies to action. Am J Ophthalmol. 1999;128(3):359-60.

7. Vitale S, Sperduto RD, Ferris FL 3rd. Increased prevalence of myopia in the United States between 1971-1972 and 1999-2004. Arch Ophthalmol. 2009;127(12):1632-9.

8. Holden BA, Fricke TR, Wilson DA, Jong M, Naidoo KS, Sankaridurg P, et al. Global prevalence of myopia and high myopia and temporal trends from 2000 through 2050. Ophthalmology. 2016;123(5):1036-42.

9. Young TL, Ronan SM, Drahozal LA, Wildenberg SC, Alvear AB, Oetting WS, et al. Evidence that a locus for familial high myopia maps to chromosome 18p. Am J Hum Genet. 1998;63(1):109-19.

10. Young TL. Dissecting the genetics of human high myopia: a molecular biologic approach. Trans Am Ophthalmol Soc. 2004;102:423-45.

11. Verhoeven VJ, Buitendijk GH, Consortium for Refractive E, Myopia, Rivadeneira F, Uitterlinden $A G$, et al. Education influences the role of genetics in myopia. Eur J Epidemiol. 2013;28(12):973-80.

12. Goldschmidt $E$, Jacobsen N. Genetic and environmental effects on myopia development and progression. Eye (Lond). 2014;28(2):126-33.

13. Shi Y, Li Y, Zhang D, Zhang H, Li Y, Lu F, et al. Exome sequencing identifies ZNF644 mutations in high myopia. PLoS Genet. 2011;7(6):e1002084.

14. Song Y, Zhang F, Zhao Y, Sun M, Tao J, Liang Y, et al. Enlargement of the axial length and altered ultrastructural features of the sclera in a mutant lumican transgenic mouse model. PLoS ONE. 2016;11(10):e0163165.

15. Tran-Viet KN, St Germain E, Soler V, Powell C, Lim SH, Klemm T, et al. Study of a US cohort supports the role of ZNF644 and high-grade myopia susceptibility. Mol Vis. 2012;18:937-44.

16. Xiang $X$, Wang T, Tong $P$, Li Y, Guo H, Wan A, et al. New ZNF644 mutations identified in patients with high myopia. Mol Vis. 2014;20:939-46.

17. Jiang D, Li J, Xiao X, Li S, Jia X, Sun W, et al. Detection of mutations in LRPAP1, CTSH, LEPREL1, ZNF644, SLC39A5, and SCO2 in 298 families with early-onset high myopia by exome sequencing. Invest Ophthalmol Vis Sci. 2014;56(1):339-45.

18. Wang H, Su S, Yang M, Hu N, Yao Y, Zhu R, et al. Association of ZNF644, GRM6, and CTNND2 genes with high myopia in the Han Chinese population: Jiangsu Eye Study. Eye (Lond). 2016;30(7):1017-22.

19. Simon JM, Parker JS, Liu F, Rothbart SB, Ait-Si-Ali S, Strahl BD, et al. A role for widely interspaced zinc finger (WIZ) in retention of the G9a methyltransferase on chromatin. J Biol Chem. 2015;290(43):26088-102.

20. Bian C, Chen Q, Yu X. The zinc finger proteins ZNF644 and WIZ regulate the G9a/GLP complex for gene repression. Elife. 2015;4.

21. Dungrawala H, Rose KL, Bhat KP, Mohni KN, Glick GG, Couch FB, et al. The replication checkpoint prevents two types of fork collapse without regulating replisome stability. Mol Cell. 2015;59(6):998-1010. 
22. Olsen JB, Wong L, Deimling S, Miles A, Guo H, Li Y, et al. G9a and ZNF644 physically associate to suppress progenitor gene expression during neurogenesis. Stem Cell Reports. 2016;7(3):454-70.

23. Huang D, Swanson EA, Lin CP, Schuman JS, Stinson WG, Chang W, et al. Optical coherence tomography. Science. 1991;254(5035):1178-81.

24. Armington JC, Bloom MB. Relations between the amplitudes of spontaneous saccades and visual responses. J Opt Soc Am. 1974;64(9):1263-71.

25. Shinar Z, Chan L, Orlinsky M. Use of ocular ultrasound for the evaluation of retinal detachment. J Emerg Med. 2011;40(1):53-7.

26. Dudea SM. Ultrasonography of the eye and orbit. Med Ultrason. 2011;13(2):171-4.

27. Pardue MT, Stone RA, luvone PM. Investigating mechanisms of myopia in mice. Exp Eye Res. 2013;114:96-105.

28. Park H, Qazi Y, Tan C, Jabbar SB, Cao Y, Schmid G, et al. Assessment of axial length measurements in mouse eyes. Optom Vis Sci. 2012;89(3):296-303.

29. Benavente-Perez A, Nour A, Troilo D. Axial eye growth and refractive error development can be modified by exposing the peripheral retina to relative myopic or hyperopic defocus. Invest Ophthalmol Vis Sci. 2014;55(10):6765-73.

30. Schmucker C, Schaeffel F. A paraxial schematic eye model for the growing C57BL/6 mouse. Vision Res. 2004;44(16):1857-67.

31. Hamidzada WA, Osuobeni EP. Agreement between A-mode and B-mode ultrasonography in the measurement of ocular distances. Vet Radiol Ultrasound. 1999:40(5):502-7.

32. Meister U, Ohnesorge B, Korner D, Boeve MH. Evaluation of ultrasound velocity in enucleated equine aqueous humor, lens and vitreous body. BMC Vet Res. 2014;10:250.

33. Zhou X, Xie J, Shen M, Wang J, Jiang L, Qu J, et al. Biometric measurement of the mouse eye using optical coherence tomography with focal plane advancement. Vision Res. 2008;48(9):1137-43.

34. Kolesnikov AV, Kefalov VJ. Transretinal ERG recordings from mouse retina: rod and cone photoresponses. J Vis Exp. 2012. https://doi. org/10.3791/3424.

35. Cermak T, Doyle EL, Christian M, Wang L, Zhang Y, Schmidt C, et al. Efficient design and assembly of custom TALEN and other TAL effectorbased constructs for DNA targeting. Nucleic Acids Res. 2011;39(12):e82.

36. Doyle EL, Booher NJ, Standage DS, Voytas DF, Brendel VP, Vandyk JK, et al. TAL effector-nucleotide targeter (TALE-NT) 2.0: tools for TAL effector design and target prediction. Nucleic Acids Res. 2012;40(Web Server issue):W117-22

37. Kasparek P, Krausova M, Haneckova R, Kriz V, Zbodakova O, Korinek V, et al. Efficient gene targeting of the Rosa26 locus in mouse zygotes using TALE nucleases. FEBS Lett. 2014;588(21):3982-8.
38. Wilkinson DG, Nieto MA. Detection of messenger RNA by in situ hybridization to tissue sections and whole mounts. Methods Enzymol. 1993:225:361-73.

39. John SW, Smith RS, Savinova OV, Hawes NL, Chang B, Turnbull D, et al. Essential iris atrophy, pigment dispersion, and glaucoma in DBA/2J mice. Invest Ophthalmol Vis Sci. 1998;39(6):951-62.

40. Brown AS, Zhang M, Cucevic V, Pavlin CJ, Foster FS. In vivo assessment of postnatal murine ocular development by ultrasound biomicroscopy. Curr Eye Res. 2005;30(1):45-51.

41. Bentley E, Miller PE, Diehl KA. Use of high-resolution ultrasound as a diagnostic tool in veterinary ophthalmology. J Am Vet Med Assoc 2003;223(11):1617-22, 599.

42. Greco A, Mancini M, Gargiulo S, Gramanzini M, Claudio PP, Brunetti A, et al. Ultrasound biomicroscopy in small animal research: applications in molecular and preclinical imaging. J Biomed Biotechnol. 2012;2012:519238.

43. Pardue MT, McCall MA, LaVail MM, Gregg RG, Peachey NS. A naturally occurring mouse model of $X$-linked congenital stationary night blindness. Invest Ophthalmol Vis Sci. 1998;39(12):2443-9.

44. Pardue MT, Faulkner AE, Fernandes A, Yin H, Schaeffel F, Williams RW, et al. High susceptibility to experimental myopia in a mouse model with a retinal on pathway defect. Invest Ophthalmol Vis Sci. 2008;49(2):706-12.

45. Park H, Tan CC, Faulkner A, Jabbar SB, Schmid G, Abey J, et al. Retinal degeneration increases susceptibility to myopia in mice. Mol Vis. 2013;19:2068-79.

46. Chakraborty R, Lacy KD, Tan CC, Park HN, Pardue MT. Refractive index measurement of the mouse crystalline lens using optical coherence tomography. Exp Eye Res. 2014;125:62-70.

47. Tkatchenko AV, Tkatchenko TV, Guggenheim JA, Verhoeven VJ, Hysi PG, Wojciechowski $R$, et al. APLP2 regulates refractive error and myopia development in mice and humans. PLoS Genet. 2015;11(8):e1005432.

48. Hudson DM, Joeng KS, Werther R, Rajagopal A, Weis M, Lee BH, et al. Posttranslationally abnormal collagens of prolyl 3-hydroxylase-2 null mice offer a pathobiological mechanism for the high myopia linked to human LEPREL1 mutations. J Biol Chem. 2015;290(13):8613-22.

49. Tejedor J, de la Villa P. Refractive changes induced by form deprivation in the mouse eye. Invest Ophthalmol Vis Sci. 2003;44(1):32-6.

50. Schaeffel F, Burkhardt E, Howland HC, Williams RW. Measurement of refractive state and deprivation myopia in two strains of mice. Optom Vis Sci. 2004;81(2):99-110.

51. Davis AM, Mao J, Naz B, Kohl JA, Rosenfeld CS. Comparative effects of estradiol, methyl-piperidino-pyrazole, raloxifene, and ICI 182780 on gene expression in the murine uterus. J Mol Endocrinol. 2008;41(4):205-17.
Ready to submit your research? Choose BMC and benefit from:

- fast, convenient online submission

- thorough peer review by experienced researchers in your field

- rapid publication on acceptance

- support for research data, including large and complex data types

- gold Open Access which fosters wider collaboration and increased citations

- maximum visibility for your research: over $100 \mathrm{M}$ website views per year

At BMC, research is always in progress.

Learn more biomedcentral.com/submissions 